\title{
Time dependent variability of QT dispersion after acute myocardial infarction and its relation to ventricular fibrillation: a prospective study
}

\author{
J D Aitchison, R W F Campbell, P D Higham
}

\begin{abstract}
Objective-To show whether increased QT dispersion on admission predicts ventricular fibrillation after acute myocardial infarction, and to determine the nature of time related changes in QT dispersion.

Design-Prospective cohort study.

Setting-Coronary care units of three teaching hospitals in Newcastle-upon-Tyne over an eight month period.

Patients-All had acute myocardial infarction according to World Health Organization criteria. Interventions-For all patients, QT dispersion (QTd) and Bazett rate corrected QTc dispersion (QTcd) were measured from a high quality 12 lead ECG recorded on admission at a paper speed of $50 \mathrm{~mm} / \mathrm{s}$. In a subset, serial ECGs were recorded regularly to show time related changes in QTcd following acute myocardial infarction.

Main outcome measures-Occurrence of ventricular fibrillation within the first 24 hours after myocardial infarction.

Results-Data collected from 201 patients, 12 of whom (6\%) developed ventricular fibrillation within 24 hours. Neither QTd nor QTcd differed between those developing ventricular fibrillation and those who did not: QTd mean (SD), 74 (24) ms (95\% confidence interval (CI) 59 to 89) v 66 (24) ms (95\% CI 62 to 70), respectively; QTcd, 86 (26) $\mathrm{ms}^{0.5}$ (95\% CI 70 to 102) v 77 (29) $\mathrm{ms}^{0.5}$ (95\% CI 72 to 82 ), respectively. Significant QTcd changes occurred early after myocardial infarction.

Conclusions-Admission QTd and QTcd do not predict ventricular fibrillation after acute myocardial infarction. There are significant changes in QTcd with time, which may account for this measured lack of correlation.

(Heart 2000;84:504-508)
\end{abstract}

Keywords: acute myocardial infarction; arrhythmia; ventricular fibrillation; QT dispersion

Many techniques have been studied in an effort to identify those patients most at risk of ventricular fibrillation. One such technique is the QT dispersion measurement, defined by Cowan and colleagues in 1988 as the difference between the maximum and minimum QT interval measurements on the standard 12 lead ECG. ${ }^{1}$ We have previously shown this simple and non-invasive measurement to be a marker for inhomogeneity of ventricular recovery. ${ }^{2}$ In that study we showed a strong $(r=0.84)$ and highly significant $(\mathrm{p}<0.001)$ correlation between QT dispersion and the dispersion of ventricular recovery time from simultaneous epicardial mapping during cardiac surgery. Similar work by Hardman and associates has confirmed the association between QT dispersion and dispersion of endocardial monophasic action potentials by catheter mapping. ${ }^{3}$

Increased QT dispersion has been shown to be associated with increased risk of cardiac death in patients with non-insulin dependent diabetes mellitus ${ }^{4}$ and those awaiting heart transplantation, ${ }^{5}$ and with risk of arrhythmia in patients with congenital long QT syndromes. ${ }^{6}$ However, there is debate over whether increased QT dispersion predicts the occurrence of primary ventricular fibrillation early after myocardial infarction. Our pilot study showed a significant difference between admission QT dispersion in patients developing ventricular fibrillation or not developing it following acute myocardial infarction (mean (SD), 105 (17) $\mathrm{ms}, 95 \%$ confidence interval (CI) 74 to $136 \mathrm{~ms}$, v 87 (15) $\mathrm{ms}, 95 \%$ CI 81 to $93 \mathrm{~ms}$, respectively). ${ }^{7}$ This has been repeated by other researchers without agreement (fig 1). In this paper we present a prospective cohort study specifically designed to test this association and we show new data to account for the lack of consensus.

The aims of the study were to show whether QT dispersion is increased in patients who develop early ventricular fibrillation following acute myocardial infarction, and to determine the nature of time related changes in QT dispersion measurements.

\section{Methods}

POWER CALCULATION

From published preliminary data we have estimated that mean (SD) admission QT dispersion is 80 (20) ms after acute myocardial infarction, which increases to 100 (20) $\mathrm{ms}$ in those developing ventricular fibrillation. ${ }^{7}$ Using an anticipated incidence of ventricular fibrillation of $7.5 \%$ in power estimates, a sample of 15 patients developing ventricular fibrillation in a population of 200 shows a $90 \%$ likelihood of 


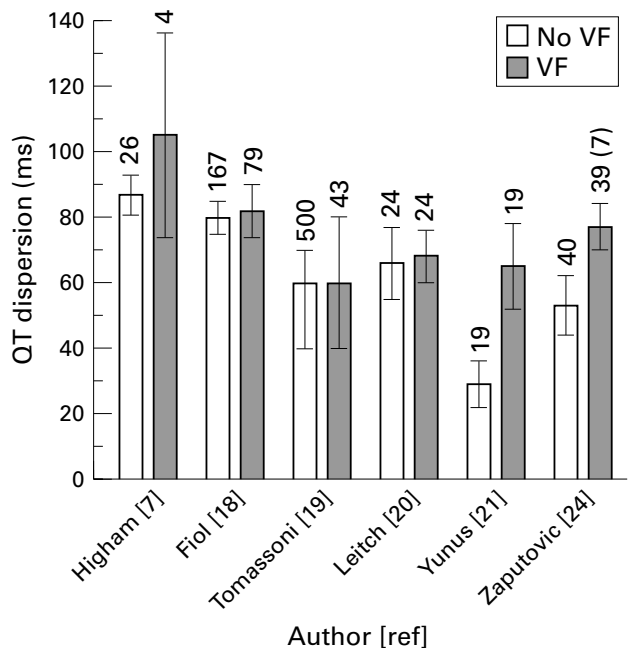

Figure 1 QT dispersion measurements in various studies. Numbers along the top show group sizes, figures in parentheses indicate number with ventricular fibrillation. Error bars show 95\% confidence intervals for the means, except for Tomassoni and colleagues ${ }^{19}$ which shows the 25th and 75 th centile measurements. VF, ventricular fibrillation.

detecting a difference of this size at a $5 \%$ probability level.

PATIENTS

This prospective study included patients with acute myocardial infarction admitted to the three coronary care units of hospitals serving the city of Newcastle-upon-Tyne during a nine month period.

INCLUSION CRITERIA

Inclusion criteria follow the World Health Organization definition of acute myocardial infarction: typical history of severe central chest pain radiating to neck or arm of duration greater than half an hour, confirmatory ECG changes of ST elevation $>2 \mathrm{~mm}$ in chest leads or $>1 \mathrm{~mm}$ in limb leads, and a rise in serum cardiac enzyme concentrations to more than twice the upper limit of normal.

DATA COLLECTION

Data collected included demographic information, detailed drug history and previous cardiovascular history, a high quality 12 lead ECG on admission (paper speed of $50 \mathrm{~mm} / \mathrm{s}$ where possible), serum potassium concentration on admission, and serial creatine kinase $\mathrm{MB}$ isoenzyme concentrations. In 12 patients, serial ECGs were recorded automatically at approximately 40 minute intervals by a Mortara ELI-100 instrument with sufficient memory for at least eight hours, and further ECGs were recorded daily for five days. Ventricular fibrillation requiring DC cardioversion was identified on the clinical monitoring equipment. Left ventricular failure was defined as typical clinical and chest $x$ ray findings of pulmonary oedema requiring treatment.

QT DISPERSION MEASUREMENT

All ECGs were analysed by a single observer blinded to patient identity, timing, and clinical information. Heart rate was measured from three consecutive RR intervals, and infarct site was defined as the area with maximum ST segment elevation. QT intervals were measured as previously documented. ${ }^{1}$ The relation between QT interval and heart rate has been extensively explored. Bazett's rate correction is the one most widely used and has been validated during the early period of acute myocardial infarction. ${ }^{8}{ }^{9}$ QT dispersion was defined as the difference between the maximum and minimum QT intervals; corrected QTc dispersion was defined as the difference between the maximum and minimum Bazett rate corrected QT intervals; and adjusted QTc dispersion was defined as the QTc dispersion divided by the square root of the number of leads in which QT intervals were measurable. The QTc standard deviation, defined as the standard deviation of the Bazett rate corrected QT intervals, represents a measure of spread which is statistically more reliable than the range, as described by Hnatkova and colleagues. ${ }^{10}$

\section{DRUG TREATMENT}

The effects of antiarrhythmic agents, anticonvulsants, ${ }^{11}$ phenothiazines, ${ }^{12}$ digoxin, ${ }^{13}$ and benzodiazepines ${ }^{14} 15$ on the ventricular myocardium are well documented. These effects may be either proarrhythmogenic or antiarrhythmogenic, depending on the circumstances, and have been shown to affect the QT interval. To avoid confounding of results because of the pharmacological effects of these drugs, electrophysiological results from patients prescribed these agents were excluded from the analysis.

INFARCT SIZE

A measure of early infarct size was made using the formula described by Clemmenson and colleagues, based on ST elevation. ${ }^{16}$ This gives a figure approximately equal to the percentage of left ventricular myocardium that is jeopardised, and has been shown to correlate well with final QRS derived infarct sizing using the Selvester technique.

QT DISPERSION PROFILES

To follow changes in QT dispersion with time, serial ECGs were analysed in 12 patients. Profiles of corrected QTc dispersion against time were plotted.

\section{STATISTICAL ANALYSIS}

For categorical data, the $\chi^{2}$ test was used. For continuous data, the two sample $t$ test was used where distributions did not differ significantly from normal. For measures of infarct size, the distributions were significantly skewed and the non-parametric Mann-Whitney U test was used. Spearman's rank correlation method was used to test for linear association. Classical analysis of variance (ANOVA) was used to test for differences between multiple groups. All tests were performed using statistics package Unistat IV on a PC compatible computer. Results are expressed as a ratio of the relevant population with percentages in parentheses or as a mean and standard deviation with $95 \%$ confidence intervals (CI) where appropriate. 
Table 1 Clinical information

\begin{tabular}{|c|c|c|c|c|}
\hline & \multicolumn{2}{|l|}{ No ventricular fibrillation } & \multicolumn{2}{|l|}{ Ventricular fibrillation } \\
\hline & ECG analysis included & ECG analysis excluded & ECG analysis included & ECG analysis excluded \\
\hline Female & $49 / 149(33 \%)$ & $11 / 40(28 \%)$ & $3 / 8$ & $1 / 4$ \\
\hline$\beta$ Blocker & $39 / 149(26 \%)$ & $8 / 40(20 \%)$ & $0 / 8$ & $1 / 4$ \\
\hline Previous myocardial infarction & $56 / 149(38 \%)$ & $20 / 40(50 \%)$ & $5 / 8$ & $2 / 4$ \\
\hline Previous coronary bypass & $5 / 149(3 \%)$ & $5 / 40(8 \%)$ & $0 / 8$ & $1 / 4$ \\
\hline Left ventricular failure & $38 / 138(28 \%)$ & $18 / 38(47 \%)$ & $4 / 8$ & $2 / 4$ \\
\hline Mortality & $10 / 149(7 \%)$ & $3 / 40(8 \%)$ & $2 / 8$ & $0 / 4$ \\
\hline Age (years) & $65(12), 63$ to 66 & $68(7), 65$ to 70 & $62(12), 49$ to 74 & $68(6), 52$ to 84 \\
\hline Infarct score $(\%)$ & $10.4(9.3), 8.9$ to 12.0 & $9.9(9.0), 7.0$ to 12.8 & $9.7(9.5), 1.7$ to 17.6 & $19.3(17.2), 0$ to 62 \\
\hline Peak CK-MB (units/l) & $83(79), 69$ to 97 & $88(83), 61$ to 116 & $82(21), 48$ to 115 & $208(80), 9$ to 406 \\
\hline Serum potassium $(\mathrm{mmol} / \mathrm{l})$ & $4.19(0.52), 4.09$ to 4.28 & $4.28(0.54), 4.13$ to 4.42 & $4.22(0.20), 4.00$ to 4.43 & $3.73(0.25), 3.11$ to 4.36 \\
\hline $\begin{array}{l}\text { Mean arterial pressure on admission } \\
(\mathrm{mm} \mathrm{Hg})\end{array}$ & $96(22), 92$ to 100 & $97(17), 92$ to 103 & 100 (18), 79 to 124 & 81 (17), 40 to 122 \\
\hline
\end{tabular}

Categorical variables are given as $\mathrm{n}(\%)$, continuous variables as mean (SD), $95 \%$ confidence interval.

\section{Results}

PATIENTS

Data were collected from 201 patients, of whom $12(6 \%)$ subsequently developed ventricular fibrillation within 24 hours of the onset of symptoms (range $0.25-23$ hours after ECG, median 1.75 hours). Clinical information is shown in table 1. Only the peak creatine kinase $\mathrm{MB}$ isoenzyme $(\mathrm{CK}-\mathrm{MB})$ differed significantly $(\mathrm{p}<0.05)$ between patients with and without ventricular fibrillation.

ECG INFORMATION

ECG information was excluded from 44 patients: eight with atrial fibrillation and 43 because of the current prescription of antiarrhythmic agents, benzodiazepines, phenothiazines, anticonvulsants, or digoxin (seven patients had both reasons for exclusion). For comparison, the results of excluded patients are also shown in table 1. ECG data are shown in table 2.

The rate corrected QTc dispersion was the originally proposed measure of QT interval variability and correlated highly with all other measures of QT interval variability: corrected QTc dispersion versus uncorrected QT dispersion, Spearman's $\rho=0.86, p<0.00005$; versus adjusted QTc dispersion, $\rho=0.99$, $\mathrm{p}<0.00005$; versus QTc standard deviation, $\rho=0.95, \mathrm{p}<0.00005$.

FACTORS AFFECTING QT DISPERSION

Neither QT dispersion nor QTc dispersion differed significantly between patients who had episodes of ventricular fibrillation and those who did not (fig 2). QTc dispersion (mean (SD) $\mathrm{ms}^{0.5}$ ) was not affected by any of the following: previous myocardial infarction (no myocardial infarction, 76 (29), 95\% CI 69 to 83, v myocardial infarction, 78 (28), 95\% CI 71 to

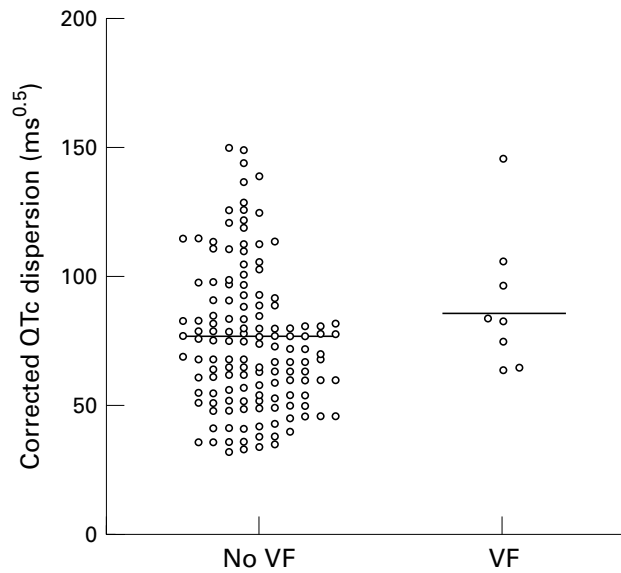

Figure 2 QTc dispersion scatterplots. Horizontal lines represent mean values for each group and a circle represents each result. VF, ventricular fibrillation.

85); infarct score (Spearman's $\rho=0.055, \mathrm{NS}$ ); infarct site (anterior, 79 (30), 95\% CI 72 to 86 , $v$ inferior, 77 (25), $95 \%$ CI 71 to 83 , $v$ lateral/ posterior, 92 (41), 95\% CI 61 to 123 , NS by ANOVA); and $\beta$ blockade (no $\beta$ blocker, 77 (29), 95\% CI 72 to $82, v \beta$ blocker, 78 (29), $95 \%$ CI 68 to 88 ).

QT DISPERSION PROFILES

Of the 12 patients studied with serial ECGs, seven had resolution of clinical symptoms and more than a $25 \%$ reduction in maximum ST segment elevation within one hour of thrombolytic treatment. This was suggestive of reperfusion, although angiographic confirmation was unavailable. Profiles of corrected QTc dispersion for all patients studied with serial ECGs are shown in fig 3, grouped by resolution or persistence of symptoms and ST segment elevation.

Table 2 Electrocardiographic information

\begin{tabular}{|c|c|c|c|c|}
\hline & \multicolumn{2}{|c|}{ No ventricular fibrillation $(n=149)$} & \multicolumn{2}{|c|}{ Ventricular fibrillation $(n=8)$} \\
\hline & Mean (SD) & $95 \% C I$ & Mean (SD) & $95 \% C I$ \\
\hline Heart rate (beats/min) & $81(22)$ & 77 to 85 & $91(20)$ & 78 to 104 \\
\hline Maximum QTc interval $\left(\mathrm{ms}^{0.5}\right)$ & $476(45)$ & 468 to 484 & $482(46)$ & 453 to 511 \\
\hline Minimum QTc interval $\left(\mathrm{ms}^{0.5}\right)$ & $399(37)$ & 393 to 405 & $396(29)$ & 378 to 414 \\
\hline Mean QTc interval $\left(\mathrm{ms}^{0.5}\right)$ & $442(37)$ & 436 to 448 & $438(30)$ & 419 to 457 \\
\hline QT dispersion (ms) & $66(24)$ & 62 to 70 & $74(24)$ & 59 to 89 \\
\hline Corrected QTc dispersion $\left(\mathrm{ms}^{0.5}\right)$ & $77(29)$ & 72 to 82 & $86(26)$ & 70 to 102 \\
\hline Adjusted QTc dispersion $\left(\mathrm{ms}^{0.5}\right)$ & $23(9)$ & 21 to 25 & $26(7)$ & 22 to 30 \\
\hline QTc standard deviation $\left(\mathrm{ms}^{0.5}\right)$ & $24(10)$ & 22 to 26 & $26(10)$ & 20 to 32 \\
\hline
\end{tabular}


Group A
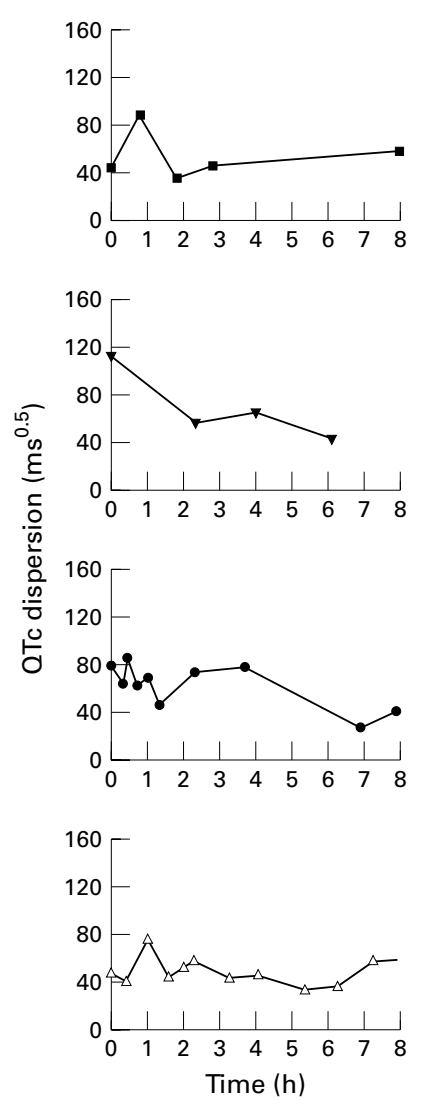
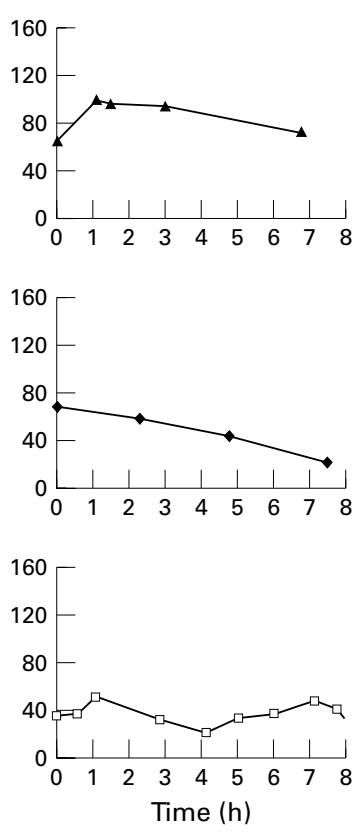

Group B
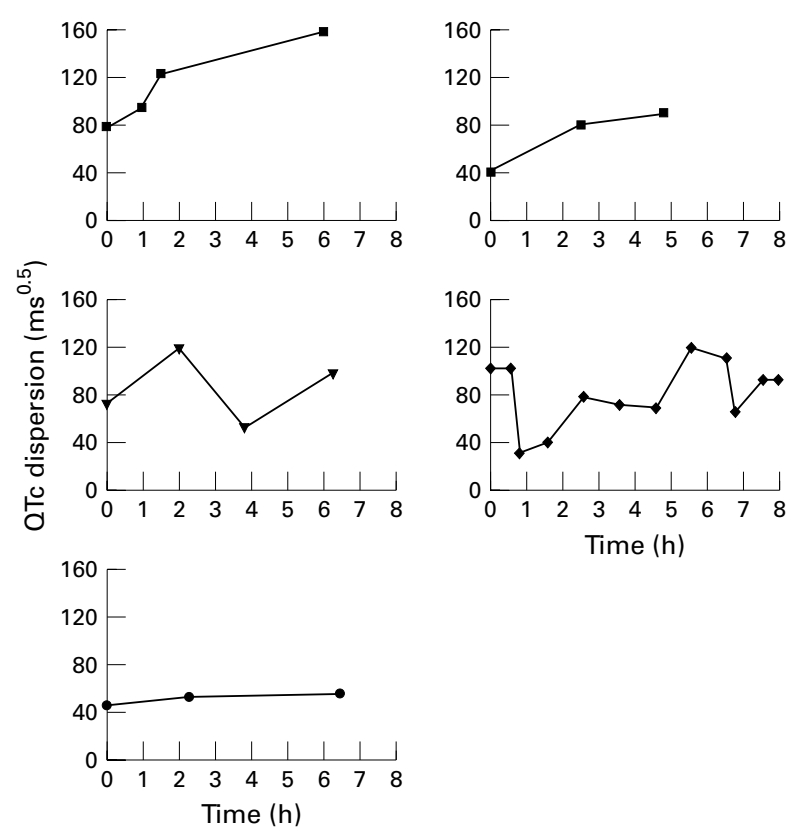

Figure 3 Examples of profiles of QTc dispersion. Each frame shows the corrected QTc dispersion profile for a single patient over the first eight hours after admission. Patients' data are grouped by resolution or persistence of symptoms and ST segment elevation following thrombolysis. The first group (group A) had resolution of symptoms and ST elevation, whereas the second (group B) did not.

Discussion

Neither QT dispersion nor QTc dispersion differed significantly between patients who had episodes of ventricular fibrillation and those who did not (table 2). Correlations between all proposed measures of QT interval variability were high and of very high significance. The very close correlation between corrected QTc dispersion and the three other measures of QT interval variability provides more rigorous statistical evidence of a spread rather than a chance finding due to outlying values. This provides further, albeit indirect, evidence that the QT dispersion measurement is an indicator of real differences in surface QT intervals, rather than a measurement artefact.

Profiles show that QTc dispersion changes from hour to hour, and this evidence of variability may well account for the lack of measured difference between patients with and without episodes of ventricular fibrillation. QTc dispersion fell in patients with resolution of symptoms and a reduction in ST elevation following thrombolysis, whereas it increased in those who did not show these features. These differences persisted, and late differences in profiles are consistent with published work showing significantly lower QT dispersion in patients with angiographically proven reperfusion at nine days after the onset of symptoms. ${ }^{17}$
Strengths of this study include its prospective nature and its specific aim to explore QT dispersion and the relation to ventricular fibrillation following acute myocardial infarction. Using a cohort study, problems with matching, as in case-control studies, are reduced. In addition, the methodology of QT dispersion used has been well described and published.

A significant limitation of the study is the small number of patients with episodes of ventricular fibrillation. The study was powered to detect a mean difference in corrected QTc dispersion of $20 \mathrm{~ms}^{0.5}$ between patients developing or not developing ventricular fibrillation. However, the observed mean difference was much smaller $\left(9 \mathrm{~ms}^{0.5}\right)$ and the study is underpowered to show a difference of this size. Nevertheless, a real but small difference of this magnitude would be unlikely to be of clinical benefit in view of the range of measured QTC dispersion measurements.

Results of the previously reported studies based on multicentre trials for other purposes are shown in fig 1 . The three largest of these $\mathrm{s}^{18-20}$ were also negative. The two positive studies both have methodological differences from the others. Yunus and colleagues measured QT dispersion from only the six anterior chest leads. ${ }^{21}$ Previous work shows that this technique is likely to affect the QT dispersion measurements significantly. ${ }^{1022}{ }^{23}$ Further, their ECGs were recorded at $25 \mathrm{~mm} / \mathrm{s}$, which has 
been shown to increase the inaccuracy of measurement. ${ }^{1}$ Zaputovic and colleagues included 32 patients with sustained ventricular tachycardia after myocardial infarction among their 39 cases. $^{24}$ Their positive result may reflect the predominant arrhythmia of ventricular tachycardia. The substrate for ventricular tachycardia is more likely to be established scar tissue and these cases may therefore be expected to differ from those with ventricular fibrillation. ${ }^{25}$ Oikarinen and associates found significant differences in QT dispersion between well matched patient groups presenting with ventricular tachycardia or ventricular fibrillation after myocardial infarction. $^{26}$

\section{CONCLUSIONS}

This study provides further evidence that admission QT dispersion does not differ between patients who do or do not subsequently develop ventricular fibrillation after acute myocardial infarction. Evidence of real time changes in QT dispersion is shown during the early phase of recovery after infarction. This variability may explain the lack of correlation between increased QT dispersion at admission and ventricular fibrillation following an infarct, despite the attractive theoretical links between dispersion of ventricular recovery and ventricular fibrillation.

We would like to acknowledge the support and enthusiasm of Professor R W F Campbell for his help in the design and analysis of the study. Professor Campbell died suddenly before this paper was completed. Thanks are due to the cardiologists of the three hospitals for permission to study their patients and the three hospitals for permission to study their patients and the
nursing staff of the three coronary care units for recording nursing staff of the three coronary care units for recording supported by the British Heart Foundation.

1 Cowan JC, Yusuff $\mathrm{K}$, Moore $\mathrm{M}$, et al. Importance of lead selection in QT measurement. Am f Cardiol 1988;61:83-7. Higham PD, Hilton CJ, Aitchison JD, et al. Does QT Higham PD, Hilton CJ, Aitchison JD, et al. Does QT

3 Hardman SMC, Walker JM, Cowie MR, et al. Can OT interval in a roving precordial ECG lead detect regional differences in endocardial action potential duration in humans? [abstract] Circulation 1995;92(suppl I):275-6

4 Naas AAO, Davidson NC, Thompson C, et al. QT and QTC dispersion are accurate predictors of cardiac death in newly diagnosed non-insulin dependent diabetes: cohort study. BMF 1998;316:745-6.

5 Pinsky DJ, Sciacca RR, Steinberg JS. QT dispersion as a marker of risk in patients awaiting heart transplantation. $\mathcal{F}$ Am Coll Cardiol 1997;29:1576-84.
6 Day CP, McComb JM, Campbell RWF. OT dispersion: an Day CP, McComb JM, Campbell RWF. QT dispersion: an indication of arrhythmia risk in patie

7 Higham PD, Furniss SS, Campbell RWF. QT dispersion and components of the QT interval in ischaemia and infarction. Br Heart f 1995;73:32-6.

8 Ahnve S. Correction of the QT interval for heart rate: review of different formulas and the use of Bazett's formula in myocardial infarction. Am Heart f 1985;109:568-74.

9 Laguna P, Caminal P, Jane R, et al. Automatic QT interval analysis in post-myocardial infarction patients. $\mathcal{F}$ Ambulat Monit 1991;4:93-111.

10 Hnatkova K, Malik M, Kautzner J, et al. Adjustment of QT dispersion assessed from 12 lead electrocardiograms for different numbers of analysed electrocardiographic leads: comparison of stability of different methods. Br Heart $\mathcal{F}$ 1994;72:390-6.

11 Iwahashi K. Significantly higher plasma haloperidol level during co-treatment with carbamazepine may herald during co-treatment with carbamazepine may her

12 Hartigan-Go K, Bateman DN, Nyberg G, et al. Concentration-related pharmacodynamic effects of thioridazine and its metabolites in humans. Clin Pharmacol Ther 1996;60:543-53.

13 Fisch C. Electrocardiography. In: Braunwald E, ed. Heart disease: a textbook of cardiovascular medicine, 5th ed. Philadelphia: WB Saunders, 1997:108-45.

14 Roelofse JA, van der Bijl P. Cardiac dysrhythmias associated with intravenous lorazepam, diazepam and midazolam during oral surgery. F Oral Maxillofac Surg 1994;52:24750.

15 Hess L, Vrana M, Vranova Z, et al. A comparison of the anti-fibrillatory effect of midazolam and flunitrazepam in acute myocardial ischaemia in the dog. Curr Med Res Opin 1987; 10:527-30

16 Clemmensen P, Grande P, Saunamaki K, et al. Effect of intravenous streptokinase on the relation between initial ST-predicted size and final QRS-estimated size of acute myocardial infarcts. 7 Am Coll Cardiol 1990;16:1252-7.

17 Moreno FL, Villanueva T, Karagounis LA, et al. Reduction in QT interval dispersion by successful thrombolytic therapy in acute myocardial infarction. Circulation 1994;90: 94-100.

18 Fiol M, Marrugat J, Bergada J, et al. QT dispersion and ventricular fibrillation in acute myocardial infarction [letter]. Lancet 1995;346:1424-5.

19 Tomassoni G, Pisano E, Gardner L, et al. QT prolongation and dispersion in myocardial ischaemia and infarction. $\mathcal{F}$ Electrocardiol 1998;30(suppl):187-90.

20 Leitch J, Basta M, Dobson A. QT dispersion does not predict early ventricular fibrillation after acute myocardial infarction. Pacing Clin Electrophysiol 1995;18:45-8.

21 Yunus A, Gillis AM, Duff HJ, et al. Increased precordial QTc dispersion predicts ventricular fibrillation during acute myocardial infarction. Am $\mathcal{f}$ Cardiol 1996;78:706-8.

22 Campbell RWF. Measurement of the QT Interval. Eur Heart f 1985;6(suppl D):81-3

23 Statters DJ, Malik M, Ward DE, et al. QT dispersion: problems of methodology and clinical significance. $\mathcal{F}$ Cardiovasc Electrophysiol 1994;5:672-85.

24 Zaputovic L, Mavric Z, Zaninovic-Jurjevic T, et al. Relationship between QT dispersion and the incidence of early venship between QT dispersion and the incidence of early veninfarction. Int f Cardiol 1997;62:211-16.

25 Jordaens LJ, Kindt H'T. Primary ventricular fibrillation: a reason to be cautious [editorial]. Eur Heart $\mathcal{F}$ 1997;18:8902.

26 Oikarinen L, Viitasalo M, Toivonen L. Dispersions of the QT interval in postmyocardial infarction patients presenting with ventricular tachycardia or with ventricular fibrillation. Am f Cardiol 1998;81:694-7. 